\title{
Proposal for Integrated Management of Verticillium Wilt Disease in Avocado Cultivar Hass Crops
}

\author{
Joaquín Guillermo Ramírez-Gil $^{1 \text {,*(D) }}$ and Juan Gonzalo Morales-Osorio ${ }^{2}$ \\ 1 Departamento de Agronomía, Facultad de Ciencias Agrarias, Universidad Nacional de Colombia sede \\ Bogotá, Carrera 45 No 26-85, Edificio 500, Oficina 228, Bogotá 111321, Colombia \\ 2 Departamento de Ciencias Agronómicas, Facultad de Ciencias Agrarias, Universidad Nacional de Colombia \\ sede Medellín, Carrera 65 No 59A-110, Bloque 11, Oficina 117-13, Núcleo El Volador, Medellín 050034, \\ Colombia; jgmoraleso@unal.edu.co \\ * Correspondence: jgramireg@unal.edu.co
}

Citation: Ramírez-Gil, J.G.;

Morales-Osorio, J.G. Proposal for Integrated Management of Verticillium Wilt Disease in Avocado Cultivar Hass Crops. Agronomy 2021, 11, 1932. https://doi.org/10.3390/ agronomy11101932

Academic Editors: Dora Batista and Marco Cristancho

Received: 31 August 2021

Accepted: 20 September 2021

Published: 26 September 2021

Publisher's Note: MDPI stays neutral with regard to jurisdictional claims in published maps and institutional affiliations.

Copyright: (C) 2021 by the authors Licensee MDPI, Basel, Switzerland. This article is an open access article distributed under the terms and conditions of the Creative Commons Attribution (CC BY) license (https:// creativecommons.org/licenses/by/ $4.0 /)$.

\begin{abstract}
The area planted with avocado crops in Colombia has been growing rapidly in recent years, especially for export varieties such as Hass. The increase in planted area coincided with increased phytosanitary problems, where pathogens such as fungi of the genus Verticillium spp. are becoming of economic importance. The objective of this study was to evaluate different control strategies for avocado wilt disease caused by Verticillium spp., under in vitro, net house, and field conditions. Strategies tested included fungicides (benomyl, azoxystrobin, captan, and carbendazim), beneficial and antagonistic microorganisms (Trichoderma sp., and Rhizoglomus fasciculatum), and physical and cultural practices such as solarization, drainage and removal of diseased tissues. Treatments T7fi (pruning-solarization-Trichoderma-mycorrhiza-sucrose-organic matter-drainage) and T8fi (fungicidepruning-solarization-Trichoderma-mycorrhiza-sucrose-organic matter-drainage), showed the greatest reduction in the area under disease progress curve and Verticillium dahliae inoculum in soil and plant tissues under field conditions. Fruit with extra quality increased $120.8 \%$ with T7fi and $108 \%$ with T8fi, compared to the control with diseased trees. The highest costs were identified for T7fi and T8fi; however, these treatments also showed the best cost/benefit relationship. Integrated approaches as in T7fi and T8fi showed the best results for Verticillium wilt control. As no fungicides of chemical synthesis are included in T7fi (pruning-solarisation-Trichoderma-mycorrhiza-sucrose-organic matterdrainage), it should be preferred to T8fi, which does include them, to avoid their negative impacts on avocado production.
\end{abstract}

Keywords: Verticillium wilt; integrated disease management; beneficial microorganism; cultural practice; disease reduction; Persea americana

\section{Introduction}

The genus of fungi Verticillium Nees comprises a cosmopolitan group of Ascomycetes, within which are classified devastating plant pathogen species that cause vascular wilt and plant death in a number of crops [1,2]. Verticillium spp. may survive in the environment for long periods of time by resistant structures and present a wide range of host plants [1-5]. Arguably, Verticillium albo-atrum Reinke and Beerthold, and Verticillium dahliae Kleb., are the species in this genus that cause the largest losses in several crops worldwide [2,6].

Control of plant diseases caused by Verticillium spp. are a challenge because infections start through the roots, making it difficult to perform detection of this causal agent and limiting the effect of most fungicides. In addition, inoculum persists for long time periods under field conditions, the pathogen exhibits a wide host range and there is a lack of resistant varieties for most crops [7-10]. Most control measures of diseases caused by Verticillium spp. are based on application of synthetic chemicals to the soil, which usually increase costs, may induce resistance in pathogen populations, show toxicity or affect the 
ozone layer as methyl bromide, and present other adverse effects on the environment and human health $[1,2,5]$.

$V$. albo-atrum and $V$. dahliae have been reported causing wilt disease on Persea americana Mill. in several countries around the world $[8,9,11,12]$. There are examples of integrated disease management programs for Verticillium wilt in crops such as tomato, olive, and many others [1,13]. However, information for avocado is scarce, and most integrated management proposals have been focused in the root rot disease caused by the oomycete Phytophthora cinnamomi Rands $[9,14]$. In general, it has been reported that pruning and other cultural practices, selection of Mexican rather than Guatemalan tolerant rootstocks, to avoid planting avocado crops on lands previously used for other crops susceptible to Verticillium spp. and to prevent the use of affected trees as a source of budwood or seeds, are agronomical practices recommended to reduce Verticillium wilt in avocado crops [15-17]. However, despite its high incidence and wide distribution, no efficient management practices are readily available [12,18].

Efficient programs for integrated disease management usually include several strategies of pathogen control. Beneficial microorganisms have proven useful for agriculture around the world. Mycorrhizas and Trichoderma spp. have been extensively studied for various aspects useful in crop production, such as fertilization, plant growth promotion and adaptation to abiotic and biotic stresses $[19,20]$. There are several reports about the effect of biological control on Verticillium spp., but in general, the results are not conclusive about the effective reduction of disease incidence and severity and on yield increase in crop fields $[1,6,21,22]$. Other practices reported as successful in reducing Verticillium spp. inoculum in avocado and others hosts are heat treatments [10], fungicide applications, plant resistance inducers, peroxygen-based disinfectants [7,13], use of manures or compost of different origins [23-26], combination of biological and chemical control measures [27], and tolerant rootstocks with different levels to avocado disease response $[15,16]$. In recent publications, the concept of "next-generation green composts" is proposed, which relates to the use of multiple sources of agricultural residues with great potential in the biocontrol and suppressive effect on soilborne plant pathogenic fungi such as $V$. dahliae $[25,26]$.

The area growing avocados in Colombia expanded $212 \%$ during the decade 2008-2018 [28]. Comparing the period from January to June of the year 2019 with the year 2020, the area growing avocado cv. Hass for export increased 34\%, with a change in value from 52 to 72 million USD of FOB price [29]. Currently, Colombia is third in area grown and fourth in avocado fruit production in the world [30].

Avocado wilt caused by Verticillium spp. has gained growing importance in Colombia during the last years up to reaching second place in diseases of biotic origin after the root rot wilt caused by P. cinnamomi [12,18]. Verticillium wilt has been detected in Colombia in the departments of Antioquia, Caldas, Cundinamarca, Risaralda, Quindío and Valle del Cauca [31]. However, studies about basic biology, epidemiology, incidence, severity, prevention, diagnosis, integrated management and economic losses of this disease are scarce [17]. As most available management strategies of avocado wilt diseases are focused on root rot caused by P. cinnamomi, but little is known about practices for other pathogens such as Verticillium spp., the present work aimed to test the in vitro activity of antagonists against this fungal pathogen and to evaluate different strategies as tools for integrated management of the avocado wilt disease caused by Verticillium spp. at net-house and field conditions. Integrated approaches, including pruning-solarization-Trichodermamycorrhiza-sucrose-organic matter-drainage, showed the best results for Verticillium wilt control, and also showed the best cost/benefit relationship.

\section{Materials and Methods}

\subsection{Experimental Site}

The present work was carried out between the years 2011-2014. Net-house average environmental conditions were temperature of $18-24{ }^{\circ} \mathrm{C}$, relative humidity of $75-95 \%$ and photosynthetically active radiation (PAR) of 650-1920 $\mu \mathrm{mol}$ photons $\mathrm{m}^{-2} \mathrm{~s}^{-1}$. Research 
under field conditions was performed in two plots planted with avocado cv. Hass trees of 6 years old at a distance of $7 \times 7 \mathrm{~m}$. Seedlings of the genotype West Indian of high altitude were used as rootstock. As farmers use plants from sexual seeds instead of a clonal rootstock, variability may be present in avocado fields, including sometimes plants of cv. Hass. Plots were located in the Department of Antioquia, Colombia in the municipalities of La Ceja, in the eastern region (5.95931 latitude-75.41777 longitude, 2387 masl), and Donmatias (6.496961 latitude-75.412118 longitude, 2213 masl) in the Northern region. Plots localization corresponded to the ecological life zone of lower montane very humid forest (vhf-LM) sensu Holdridge. Edaphoclimatic details are shown in Supplementary Material (Table S1 and Figure S1A).

\subsection{Plant Material for Evaluation under Net-House Conditions}

Avocado seeds of cv. Hass were collected from a healthy tree and disinfested; then, a pre-germination treatment was applied, and they were sown in autoclaved quartz. When seedlings exhibited five fully expanded leaves and the secondary root system was well developed, cotyledons were excised to promote root growth. Seedlings were then transferred to plastic pots containing $2 \mathrm{~kg}$ of soil (Andisol from the municipality of El Peñol, Antioquia, Colombia, autoclaved at $0.1 \mathrm{MPa}$ and $121^{\circ} \mathrm{C}$ for 2 cycles of $1 \mathrm{~h}$ each). Details of soil parameters are shown in supporting information 1 . Avocado seedlings were kept under net-house conditions at $50 \%$ of the maximum soil moisture retention capacity until further use.

\subsection{Microorganism Isolation and Identification}

$V$. dahliae strains were obtained from the collection kept at "Universidad Nacional de Colombia branch Medellín" (Vert1, Vert2, Vert3, and Vert4), which were morphologically and molecularly identified [12]. $V$. dahliae strains were used because they were the prevalent species under field conditions. Trichoderma sp. TAF22, TC2 and TC3, morphotypes were isolated from the rhizosphere of healthy and well developed avocado trees [16] Rhizoglomus fasciculatum (Thaxt.) C. Walker and A. Schüßler (syn. of Rh. fasciculatum (Thaxt.) Sieverd., G.A. Silva and Oehl.) mycorrhizal fungal strain was kindly provided by professor Dr. Walter Osorio from the microorganisms bank at Laboratorio of Microbiología Ambiental del Suelo, from "Universidad Nacional de Colombia branch Medellín".

\subsection{In Vitro Assays}

In vitro assays were performed to determine the inhibitory capacity of fungicides of chemical synthesis and Trichoderma sp. Morphotypes. An r-mycelium plug taken from the first $5 \mathrm{~mm}$ of the edge of an actively growing $V$. dahliae strain in sterile potato dextrose agar (PDA) medium culture (PDA, Difco, Lawrence, KS, USA) was transferred to fresh PDA in a side of a Petri dish, and on the other side, a plug of $5 \mathrm{~mm}^{2}$ taken from an actively growing Trichoderma sp. strain in PDA was placed. The doses of fungicides under in vitro condition were designed based on previous tests associated with the use of different molecules to control of Verticillium species. Fungicides of chemical synthesis tested were Benomyl $\left(0.05 \%\right.$ of $500 \mathrm{~g}$ of active ingredient (a.i.) $\mathrm{kg}^{-1}$, Dupont, Bogotá, Colombia) [32], Azoxystrobin (40 mL L ${ }^{-1}$ of 23.2 a.i. $\mathrm{kg}^{-1}$, Syngenta, Bogotá, Colombia) [13], Captan (10 mg L (1 $^{-1}$ of $500 \mathrm{~g}$ a.i. $\mathrm{kg}^{-1}$, Adama, Barranquilla, Colombia), and Carbendazim (20 mL L ${ }^{-1}$ of $500 \mathrm{~g}$ a.i. $\mathrm{kg}^{-1}$, Vecol, Bogotá, Colombia) [33]. Tests were developed on PDA, supplemented with the different fungicides. In each treatment, a plug of mycelium of $5 \mathrm{~mm}^{2}$ taken from an actively growing $V$. dahliae strain in PDA was transferred to fresh PDA amended with each corresponding fungicide. Radial growth rate was measured as the response variable of the $V$. dahliae strain tested. It was measured daily for 20 days using a caliper (Mitutoyo Digimatic Caliper, Sendai, Japan). Experiments were repeated twice through time. 


\subsection{Net-House Tests}

\subsubsection{Pathogenicity Tests on Avocado cv. Hass Plants}

$V$. dahliae strains (Vert1, Vert2, Vert3, and Vert4), were grown on PDA medium as described before. Conidia were collected in sterile distilled water and concentration adjusted to $1 \times 10^{5}$ conidia $\mathrm{mL}^{-1}$. Then, $100 \mathrm{~mL}$ of conidia suspension were added, distributed in four equidistant points to each pot containing an avocado plant. Soil humidity was increased to $70 \%$ of the maximum retention capacity to favor disease development. Aggressiveness of each isolate was determined as the area under disease progress curve (AUDPC) [34] using a specific disease scale developed and calibrated for Verticillium spp. infecting avocado in Colombia [35].

2.5.2. Effect of Fungicides, Solarization, and Inoculation with Beneficial Microorganism (Trichoderma sp. and $R$. fasciculatum) on $V$. dahliae inoculum)

Two experiments were designed with the objective to know the effect on soil infected with $V$. dahliae. of the addition of fungicides and solarization. First, $100 \mathrm{~mL}$ of a suspension of $1 \times 10^{5}$ conidia $\times \mathrm{mL}^{-1}$ of $V$. dahliae (Vert 2 ) were added to each pot containing $2 \mathrm{~kg}$ of soil. Then, avocado plants were planted into inoculated pots and were maintained until the plants died to produce inoculum. Afterward, $500 \mathrm{~mL}$ of a solution with each fungicide, diluted in sterile distilled water at doses described previously, were applied into the infested soil, and distributed through time in three applications at days 5, 10, and 15. For solarization, a layer of $10 \mathrm{~cm}$ thick soil was covered with plastic film (transparent, caliber 4) for 40 days. In addition, temperature during solarization was measured using a digital sensor coupled with communication and storage of information, assembled in an Arduino Uno plate [36]. Solarized soil was then used to fill the pots for performing the experiments. Each experimental unit consisted of a pot with $2 \mathrm{~kg}$ of soil, three experimental units were used per treatment in a completely randomized experimental design. All experiments were repeated twice through time.

In each experiment, quantification of $V$. dahliae inoculum was performed by serial dilutions in sterile distilled water obtained from 15 random samples of $10 \mathrm{~g}$ of soil each, to recover structures of $V$. dahliae (Vert 2) that were quantified in PDA medium acidified with lactic acid (PDA-A) supplemented with streptomycin $\left(100 \mu \mathrm{g} \mathrm{L}^{-1}\right)$. Inoculum quantification was performed every five days after treatment application and during 40 days.

Based on previous tests, a new experiment was proposed to be implemented for plants under net-house conditions. Treatments were (where "nh" stands for net-house conditions): a non-inoculated control of healthy plants (nh-healthy control); a control of plants inoculated with $V$. dahliae (Vert 2) (nh-diseased control); T1nh (fungicide): Verticilliuminoculated plants + benomyl applied in drench; T2nh (Trichoderma): Verticillium-inoculated plants + inoculation with Trichoderma sp. TC2; T3nh (mycorrhiza): Verticillium-inoculated plants + inoculation with $R$. fasciculatum; T4nh (fungicide-Trichoderma-mycorrhiza): Verticillium-inoculated plants + simultaneous application of T1nh + T2nh + T3nh; and T5nh (Trichoderma-mycorrhiza): Verticillium-inoculated plants + simultaneous application of $\mathrm{T} 2 \mathrm{nh}+\mathrm{T} 3 \mathrm{nh}$. In each treatment, $V$. dahliae inoculum were added to the soil as described before, and Trichoderma sp. and R. fasciculatum were applied following the method described in [14]. Fungicide was diluted in sterile distilled water at the dose recommended by the manufacturer and $200 \mathrm{~mL}$ of fungicide solution $\times$ plant $^{-1}$ were applied directly to the soil when plants showed the degree one of disease scale developed for Verticillium spp. [35]. Aall treatments under net-house conditions may be observed in Supplementary Material Table S2.

Disease severity was measured weekly during 120 days using the aforementioned scale of severity [35], and with the obtained data, the AUDPC was calculated for each treatment [34]. At the end of the experiment, $V$. dahliae inoculum were quantified in plant tissues and soil. For plant tissues, 15 random samples of $10 \mathrm{~g}$ of root and stem pieces were processed according to assay reported to this microorganisms [12,36]. Briefly, tissues were macerated and serial dilutions were prepared in sterile distilled water. Dilutions 
were placed and dispersed in Petri dishes containing PDA media, incubated, and colony forming units (UFC) were quantified. The procedure described before was followed for quantification of $V$. dahliae in soil. Total inocula of $V$. dahliae were considered as the sum of inocula from soil plus inocula from plant tissues. Mycorrhizal colonization was calculated using the line interception method [37]. Avocado roots were collected and washed with tap water, then were bleached in $\mathrm{KOH}(10 \%)$ for $24 \mathrm{~h}$ [38], followed by alkaline immersion $\left(0.5 \% \mathrm{NH}_{4} \mathrm{OH}\right.$ and $0.5 \% \mathrm{H}_{2} \mathrm{O}_{2} \mathrm{v} / \mathrm{v}$, in aqueous solution) for $30 \mathrm{~min}$. Mycorrhizal hyphae were visualized by staining with trypan blue (0.025\%) [39].

Additionally, to verify the effect of fungicides on Trichoderma sp. and R. fasciculatum populations, an experiment was developed in pots using the same procedure described before. Plants of Leucaena leucocephala Lam, which is an indicator of mycorrhization, were germinated on sterile quartz until three fully developed leaves were observed and then were transferred to previously prepared pots. $R$. fasciculatum and Trichoderma sp. were inoculated as described before. Tested fungicides were applied at each dose at 10 and 30 days after microbe inoculation into the soil. Mycorrhizal colonization and quantification of AVM propagules and Trichoderma sp. CFUs, were evaluated 40 days after the inoculation of microorganisms following procedures already described. Pots containing plants of L. leucocephala inoculated with both microorganisms were used as a negative control. All results may be observed in Figure S2.

\subsection{Assays under Field Conditions}

Based on data obtained from experiments performed under in vitro and net-house conditions described before, the following treatments were evaluated under field conditions (where "fi" stands for field conditions): fi-healthy control: non-inoculated control of healthy plants; fi-diseased control: plants infected with V. dahliae; T1fi (fungicide): Verticillium-infected plants + application of fungicide in drench $(3000 \mathrm{~mL}$ of benomyl solution $\left(0.05 \%\right.$ of $500 \mathrm{~g}$ a.i. $\left.\left.\mathrm{kg}^{-1}\right)\right)$ in rotation with azoxystrobin $\left(3000 \mathrm{~mL}\right.$ of $40 \mathrm{~mL} \mathrm{~L}{ }^{-1}, 23.2$ a.i.), sprayed around a diameter of $2 \mathrm{~m}$ from the base of the stem of the avocado trees; T2fi (pruning): Verticillium-infected plants + sanitary pruning (trimming all symptomatic and necrosed tissues); T3fi (solarization): Verticillium-infected plants + solarization covering a diameter of two meters around the base of the stem with a plastic film (caliber \# 4); T4fi (Trichoderma-mycorrhiza-sucrose): Verticillium-infected plants + soil inoculation with the following mixture: Trichoderma sp. morphotype TC2 (1000 mL of a solution in sterile distilled water at a concentration of $1 \times 10^{5}$ conidia per plant) plus $R$. fasciculatum ( $1 \mathrm{~kg}$ of roots and soil with $R$. fasciculatum, prepared from mycorrhiza-associated maize plants, adjusted at a concentration of 45 propagules per $g$ of soil), and the addition of $1000 \mathrm{~g}$ of sucrose diluted in sterile distilled water $(1: 1 \mathrm{p}: \mathrm{v})$ as a source of energy $[40,41]$; T5fi (organic matter): Verticillium-infected plants + application of $10 \mathrm{~kg}$ of compost in a diameter of two meters around of the base of the stem, made with bovine manure, mineral amendment $\left(\mathrm{P}_{2} \mathrm{O}_{5}, 13 \% ; \mathrm{CaO}, 25 \% ; \mathrm{MgO}, 7 \% ; \mathrm{S}, 10 \% ; \mathrm{SiO}_{2}, 6 \%\right)$ and a layer of $15 \mathrm{~cm}$ thick of homogenized plant residues and mushroom residues (1:1) [14]; T6fi (drainage): Verticillium-infected plants + a drainage network around the base of the stem consisting of a crescent-shape ditch in the upper zone of the slope $(10 \mathrm{~cm}$ wide and $10 \mathrm{~cm}$ depth $)$ and a drain in the lower zone of the slope $(20 \mathrm{~cm}$ length, $10 \mathrm{~cm}$ wide and $30 \mathrm{~cm}$ depth); T7fi (pruning- solarization-Trichoderma-mycorrhiza-sucrose-organic matter-drainage): Verticillium-infected plants + integrated management proposal without fungicide consisting of simultaneous application of T2fi + T3fi + T4fi + T5fi + T6fi; and T8fi (fungicidepruning-solarization-Trichoderma-mycorrhiza-sucrose-organic matter-drainage): Verticillium-infected plants + integrated management proposal with fungicide consisting of simultaneous application of T1fi + T2fi + T3fi + T4fi + T5fi + T6fi.

A scheme summarizing drainage treatment under field conditions may be observed in Figure S3.

Treatments were applied in 7 year old plants showing symptoms corresponding to scale 2 of wilt disease under field conditions, caused by $V$. dahliae [35]. V. dahliae was 
verified using a polyphasic approach, which included plant symptomatology, morphology and molecular characterization [12]. Treatments for soil solarization and drainage were performed at the initial stage of the experiment. Soil solarization was applied during 40 days. Fungicides were applied when plants showed symptoms corresponding to a value above 2 of the scale of the wilt disease. In T1fi, three applications of fungicide were performed. For T8fi, one application of fungicide was made before solarization and inoculation with $R$. fasciculatum and Trichoderma sp. Incorporation of compost, mineral amendment and plant and mushroom residues were performed every six months for two years. For pruning of affected plants, permanent monitoring for symptomatic and necrosed tissues was carried out. Benefic microorganisms and the energy source were applied at the beginning of the experiment and one year later. In treatments in which microorganism application was combined with solarization, microbes were applied after soil solarization.

Disease severity was measured, and the AUDPC calculated every two months for two years [34]. At the end of the experiment, inoculum of Trichoderma sp., mycorrhizal colonization and inoculum of $V$. dahliae were quantified following procedures described above. During the whole period of evaluation, the accumulated fruit production, the percentage of fruit of extra quality and the unitary cost of each treatment were determined. Using these parameters, the cost/benefit ratio was calculated [14].

All treatments under field conditions may be observed in Table S3.

\subsection{Experimental Design and Statistical Analyses}

An in vitro experimental unit was a Petri dish, 10 replicates were performed per treatment and the experiments were repeated twice through time using a completely randomized experimental design. At net-house, three plants were an experimental unit, five replicates were performed per each treatment and the experiments were repeated twice through time using a completely randomized experimental design. Under field conditions, three 7 year old plants were used as an experimental unit, with three replicates per treatment and using a randomized block experimental design (consisting of each plot), where the primary factor were the treatments and the secondary factor was the terrain slope classified in three arbitrary categories (0-10, 10.1-25 and >25.1). Under Colombian conditions there are two harvesting seasons per year; therefore, each year tested corresponded to two harvesting seasons for a total of four harvesting seasons. Data homoscedasticity and normality were determined using the Levene and KolmogorovSmirnov tests, respectively. Means were compared by analysis of variance (ANOVA) followed by the Tukey test $(p \leq 0.05)$. All statistical analyses were performed using the computational package $\mathrm{R}$.

\section{Results}

\subsection{In Vitro Assays}

All fungicides tested inhibited more than 95\% the growth of $V$. dahliae $(p<0.05)$ (Figure 1A). Similarly, but to a lesser extent, all isolates of Trichoderma sp. significantly reduced the growth of all $V$. dahliae isolates tested $(p<0.05)$, when compared to the control (Figure 1B). Isolate TC2 showed the best effect in decreasing the growth of $V$. dahliae isolates Vert1 (47.7\%), Vert2 (44.5\%), Vert3 (55.1\%) and Vert4 $(41.19 \%)(p<0.05)$ (Figure 1B). 

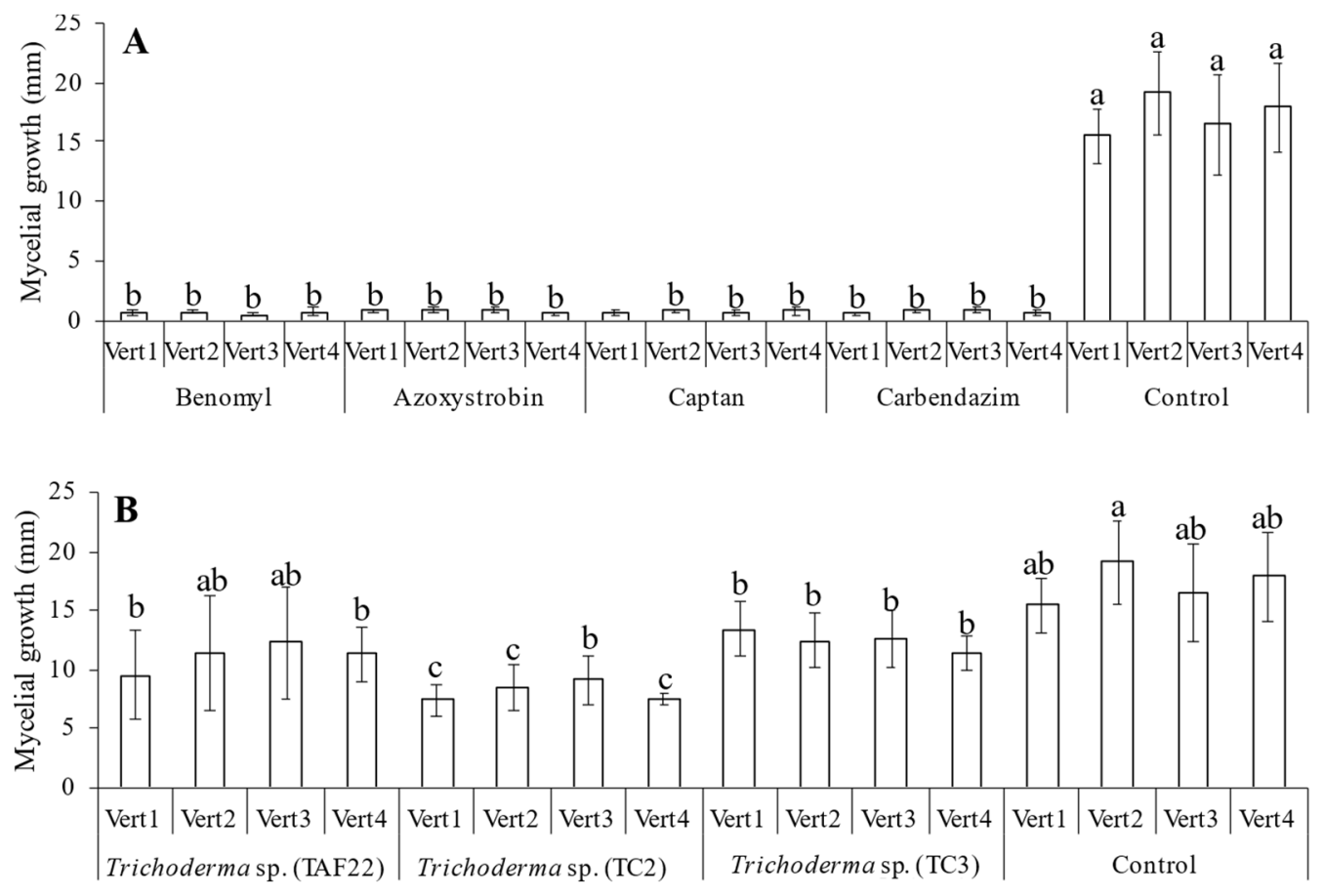

Treatment

Figure 1. Effect of different treatments on in vitro growth of Verticillium dahliae. (A): effect of different chemical fungicides on growth of Verticillium dahliae. (B): effect of different strains of Trichoderma sp., on growth of Verticillium dahliae. Vert1-4 = code of strain of Verticillium dahliae. TAF22, TC2 and TC3 = code of morphotypes of Trichoderma sp. Error bars represent the confidence interval of the mean, validated by Tukey mean separation test. Equal letters indicate that there are no significant differences $(p>0.05)$.

\subsection{Net-House Assays}

Isolate Vert2 showed the highest aggressiveness of all $V$. dahliae isolates $(p<0.05)$, exhibiting AUDPC values higher than isolates Vert3 (19.6\%) and Vert1 (24.4\%) (Figure 2A). All fungicides evaluated and solarization decreased $V$. dahliae inoculum in soil, compared to the control $(p<0.05)$; however, none completely eradicated this fungus. Benomyl showed the best effect followed by Azoxystrobin, Carbendazim and Captan $(p<0.05)$ (Figure 2B). The effect of solarization at 5, 10 and 15 days after application of treatments, was inferior to reduction observed for fungicides $(p<0.05)$; nevertheless, for a longer period of time (i.e., $>15$ days), solarization exhibited a higher reduction of $V$. dahliae inoculum than Azoxystrobin, Carbendazim or Captan, and similar to Benomyl $(p>0.05)$ (Figure 2B). It is apparent that under solarization, fluctuations of $V$. dahliae inoculum through time, coincided with fluctuations in the maximum temperature, with lower quantities of inoculum observed when the temperature was above $35^{\circ} \mathrm{C}$ (Figure 2B and Figure S1B).

All treatments significantly reduced the AUDPC caused by $V$. dahliae $(p<0.05)$. Benomyl, when applied in drench alone (T1nh) or in combination with $R$. fasciculatum and Trichoderma sp. (T4nh), showed the best reduction of AUDPC values: 65.5\% (T1nh) and $68 \%$ (T4nh), respectively, compared to the control inoculated with $V$. dahliae. T5nh (R. fasciculatum plus Trichoderma sp.,), T2nh (Trichoderma sp.,) and T3nh (R. fasciculatum), exhibited reductions in the AUDPC values of 51.4, 44.5 and 30.62\%, respectively (Figure 3A). $V$. dahliae inoculum levels measured for each treatment coincided with AUDPC values $(p<0.05)$. For T1nh, T4nh, T5nh and T2nh, reduction in AUDPC values of 55.1, 51.7, 41.3 and $24.1 \%$, respectively, was quantified compared to the control inoculated with $V$. dahliae. For T3nh, no significant differences were observed when compared to the control inoculated with $V$. dahliae $(p>0.05)$ (Figure 3B). 

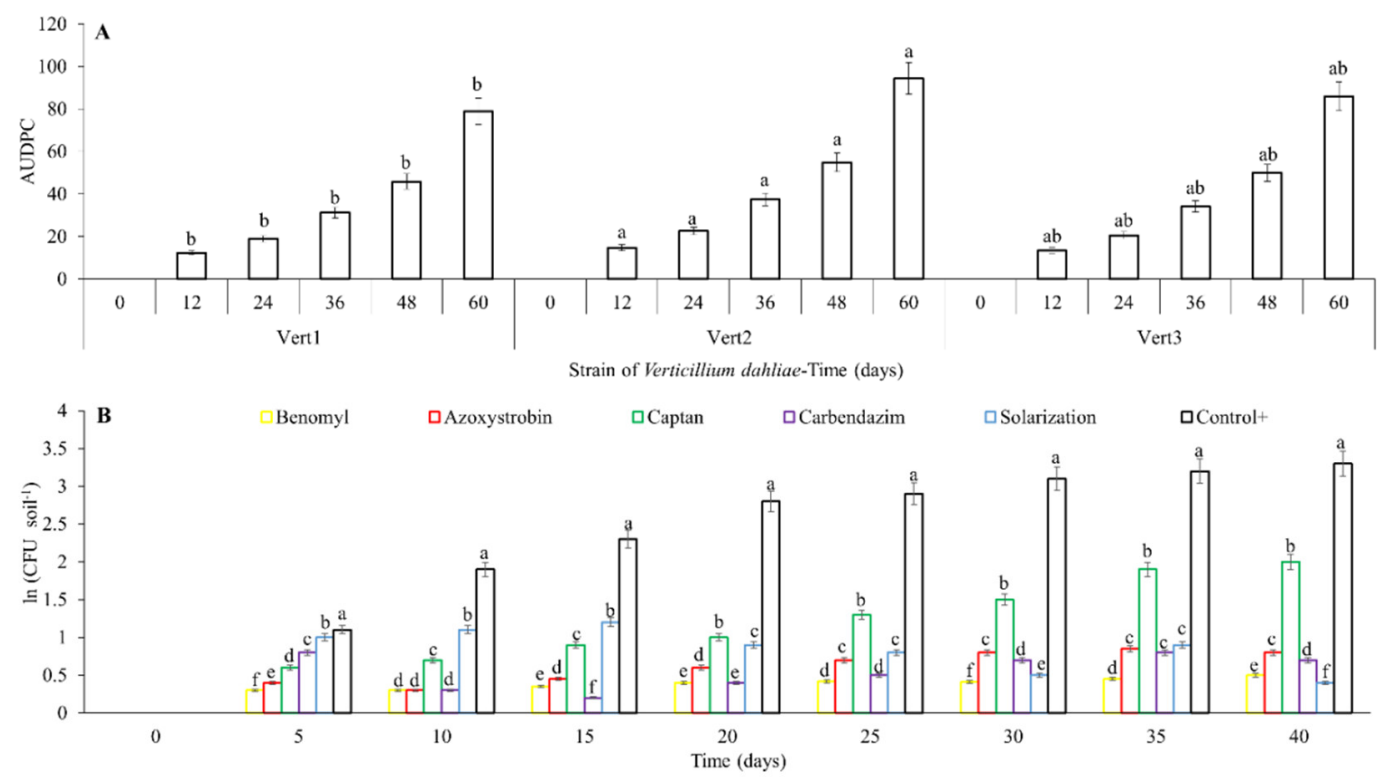

Figure 2. AUDPC and inoculum quantification for management practices of Verticillium dahliae in Hass avocado under net-house conditions. (A): evaluation of strain aggressiveness of $V$. dahliae on Hass avocado. (B): effect of chemical fungicide and solarization on soil inoculum of $V$. dahliae. Error bars represent the confidence interval of the mean, validated by Tukey mean separation test. Equal letters indicate that there are no significant differences $(p>0.05)$.
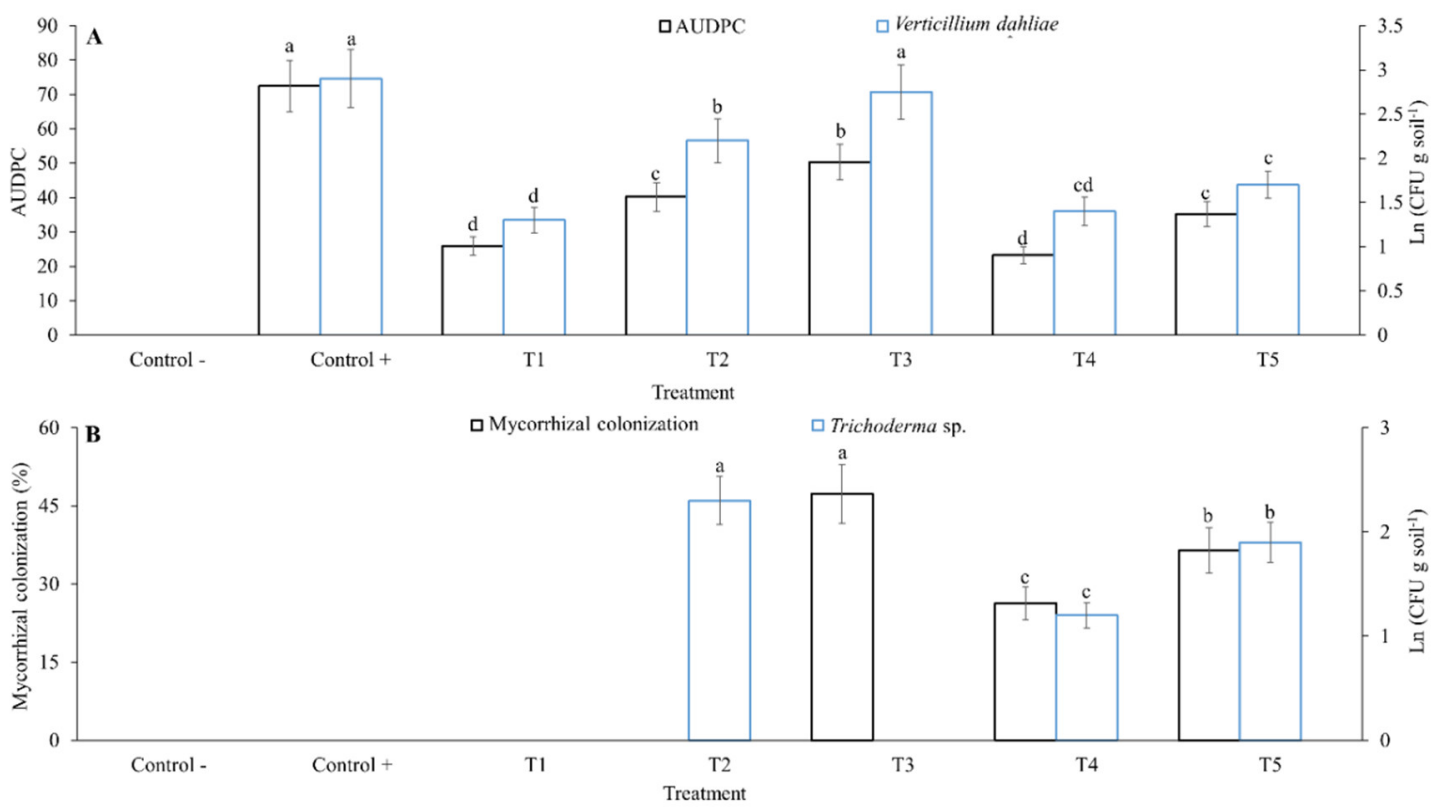

Figure 3. Effect of management practices of Verticillium dahliae in Hass avocado under net-house conditions on beneficial population of soil microorganism and reduction of AUDPC. (A): area under disease progress curve (AUDPC) of wilt disease under different treatments. (B): mycorrhizal colonization and inoculum of Trichoderma sp. quantified under different treatments for wilt disease. Error bars represent the confidence interval of the mean, validated by Tukey mean separation test. Equal letters indicate that there are no significant differences $(p>0.05)$. Treatments were (where "nh" stands for net-house conditions): a non-inoculated control of healthy plants (nh-healthy control); a control of plants inoculated with $V$. dahliae (Vert 2) (nh-diseased control); T1nh (fungicide): Verticillium-inoculated plants + benomyl; T2nh (Trichoderma): Verticilliuminoculated plants + inoculation with Trichoderma sp.; T3nh (mycorrhiza): Verticillium-inoculated plants + inoculation with R. fasciculatum; T4nh (fungicide-Trichoderma-mycorrhiza): Verticillium-inoculated plants + simultaneous application of T1nh + T2nh + T3nh; and T5nh (Trichoderma-mycorrhiza): Verticillium-inoculated plants + simultaneous application of $\mathrm{T} 2 \mathrm{nh}+\mathrm{T} 3 \mathrm{nh}$. 
As expected, Trichoderma sp. and mycorrhizal colonization values were higher $(p<0.05)$ in the treatments where individual application of these microorganisms was performed (T2nh and T3nh, respectively). In contrast, combined application of these microorganisms with a fungicide (T4nh) exhibited reduction of these variables $47.8 \%$ and $52.7 \%$, respectively, when compared to T2nh (Figure S2). Similarly, application of Trichoderma sp. and mycorrhiza without fungicide (T5nh), reduced values of these variables $34.4 \%$ and $23 \%$, respectively, compared to values obtained in T3nh (Figure 3B).

\subsection{Assays under Field Conditions}

$V$. dahliae caused plant death when no control measures were applied as evidenced in the diseased control treatment, confirming pathogenicity of isolates. A similar effect was observed in the treatment where only drainages were made (T6fi). In the other treatments, no plant death was observed and their effects were classified into two groups. In the first group, composed by T1fi (fungicides Benomyl and Azoxystrobin) and integrated management proposals without (T7fi) or with fungicides (T8nh), disease severity was always below a value of 3 in the scale used (Figure 4A). In the second group, composed by treatments T5fi (mineral amendment, bovine manure and plant and mushroom residues), T4fi (Trichoderma sp., R. fasciculatum and energy source), T3fi (solarization) and T2fi (pruning), disease severity was between values of 3 and 4 according to the scale used (Figure $4 \mathrm{~A}$ ).

All treatments evaluated, with the exception of drainage alone (T6fi), showed significant reduction of AUDPC and $V$. dahliae inoculum in soil and plant tissues $(p<0.05)$, compared to the diseased control. Integrated management proposals (T7fi and T8fi) showed the greatest reduction in AUDPC and $V$. dahliae inoculum in soil and plant tissues, followed by treatments T1fi, T5fi, T2fi, T3fi and T4fi, which reduced the AUDPC value by 80.3, 79.1, $62.3,50.3,42.2,35.7$ and $27.7 \%$, respectively; and reduced $V$. dahliae inoculum by $76.9,69.2$, $56.4,53.8,48.7,51.28$ and $41.0 \%$, respectively (Figure $4 \mathrm{~B}$ ).

Accumulated production of fruit and the amount of fruit classified in quality "extra", significantly varied for all treatments applied $(p>0.05)$. As expected, the highest values for these variables were found in the healthy control, and the lowest values were observed in the inoculated and not treated control $(p<0.05)$. Diseased plants showed a reduction in fruit production of $54.2 \%$ and a reduction in the extra quality of fruit of $59.6 \%$, when compared to healthy plants. In the integrated management treatments (T7fi and T8fi), fruit production increased $26.9 \%$ (T7fi) and $25.7 \%$ (T8fi), and fruit with extra quality increased $120.8 \%$ (T7fi) and $108 \%$ by (T8fi), compared to the control with diseased trees. In treatments T1fi, T5fi, T2fi, T3fi and T4fi, avocado trees increased fruit production in 20.1, 15.9, 14.5, 12.3 and $9.9 \%$, respectively, and increased the percentage of fruit classified as extra quality in $82.4,64,71.2,52$ and $36 \%$, respectively, compared to the diseased control (Figure 5A).

Similar results to those observed in the net-house assays for mycorrhizal colonization and the amount of inoculum of Trichoderma sp., were observed in field conditions $(p<0.05)$ (Figure 5B).

The highest costs among all treatments tested for $V$. dahliae management were identified for the integrated management proposals T7fi and T8fi; however, these treatments also showed the best cost/benefit relationship (Table 1). Likewise, in the evaluated treatments, there were large differences in production and losses per year (Table 1). In percentage terms, the treatment with infected plants $($ Control + ) presented a reduction of $212.9 \%$ with respect to the non-diseased control (Control-), contrasting with the integrated management proposal (T8fi), with a reduction of $18 \%$. 
Table 1. Costs associated with Verticillium dahliae in avocado cv. Hass crops in Antioquia department, Colombia.

\begin{tabular}{|c|c|c|c|c|c|c|c|}
\hline \multirow{2}{*}{ Treatment } & \multirow{2}{*}{$\begin{array}{c}\text { Variable Unit } \\
\text { Cost for Tree/Two } \\
\text { Years }{ }^{a, b}\end{array}$} & \multicolumn{2}{|c|}{$\begin{array}{l}\text { Cost of Production of } \\
1 \mathrm{~kg}^{\mathrm{c}}\end{array}$} & \multicolumn{2}{|c|}{ Yield and Reduction } & \multirow{2}{*}{$\begin{array}{c}\text { Cost/Benefit } \\
\text { Ratio }\end{array}$} & \multirow{2}{*}{$\begin{array}{c}\text { Cost/Benefit Ratio } \\
\text { as \% Respect to } \\
\text { Control }^{\text {e }}\end{array}$} \\
\hline & & Fixed ${ }^{a, b}$ & Variable $^{a, b}$ & $\begin{array}{c}\text { Yield } \\
\text { (kg/tree) }\end{array}$ & $\begin{array}{l}\text { Reduction } \\
(\%)^{d}\end{array}$ & & \\
\hline Control+ & 0.0 & 0.35 & 0.32 & 20.9 & 212.9 & 0.95 & 1 \\
\hline T1fi ${ }^{f}$ & 5.2 & 0.35 & 0.65 & 39.5 & 65.5 & 3.30 & 347 \\
\hline $\mathrm{T} 2 \mathrm{fi}$ & 2.5 & 0.35 & 0.50 & 28.2 & 131.9 & 2.00 & 210 \\
\hline T3fi & 4.5 & 0.35 & 0.45 & 25.0 & 161.6 & 1.50 & 157 \\
\hline T4fi & 4.2 & 0.35 & 0.40 & 28.1 & 132.7 & 1.65 & 175 \\
\hline T5fi & 6.5 & 0.35 & 0.42 & 31.4 & 108.2 & 2.2 & 231 \\
\hline T6fi & 2.2 & 0.35 & 0.40 & 25.9 & 152.5 & 1 & 1.05 \\
\hline T7fi & 19.9 & 0.35 & 0.90 & 50.3 & 30.0 & 4.5 & 473 \\
\hline T8fi & 25.1 & 0.35 & 0.95 & 55.4 & 18.0 & 4.7 & 494 \\
\hline Control- & 0.0 & 0.35 & 0.32 & 65.4 & na & na & na \\
\hline
\end{tabular}

${ }^{a}$ Variable cost associated with the management treatment of diseased plants infected with Verticillium dahliae. ${ }^{\mathrm{b}}$ Value given in constant USD for 23 December of 2014 with an exchange rate of COP 2392.4 per USD 1. ${ }^{c}$ The absorption costing method was used for calculating the cost of each treatment. ${ }^{d}$ The production reduction was determined based on the yield of each treatment compared to the control—(healthy plants). ${ }^{\text {e }}$ The cost/benefit ratio method was applied to calculate farm income related to each treatment applied, quantified by dividing the cost/benefit ratio of each treatment by the cost/benefit ratio of $\mathrm{T} 0$, multiplied by $100 .{ }^{\mathrm{f}}$ fi stands for treatments under field conditions. The following treatments were evaluated under field conditions (where "fi" stands for field conditions): fi-healthy control: non-inoculated control of healthy plants; fi-diseased control: plants infected with $V$. dahliae; T1fi (fungicide): Verticillium-infected plants + application of fungicide in drench (benomyl in rotation with azoxystrobin); T2fi (pruning): Verticillium-infected plants + sanitary pruning; T3fi (solarization): Verticilliuminfected plants + solarization; T4fi (Trichoderma-mycorrhiza-sucrose): Verticillium-infected plants + soil inoculation with Trichoderma sp. and R. fasciculatum; T5fi (organic matter): Verticillium-infected plants + application of compost; T6fi (drainage): Verticillium-infected plants + a drainage network around the base of the stem; T7fi (pruning-solarization-Trichoderma-mycorrhiza-sucrose-organic matter-drainage): Verticillium-infected plants + integrated management proposal without fungicide consisting of simultaneous application of T2fi + T3fi + T4fi + T5fi + T6fi; and T8fi (fungicide-pruning-solarization-Trichoderma-mycorrhiza-sucrose-organic matter-drainage): Verticillium-infected plants + integrated management proposal with fungicide consisting of simultaneous application of T1fi + T2fi + T3fi + T4fi + T5fi + T6fi.

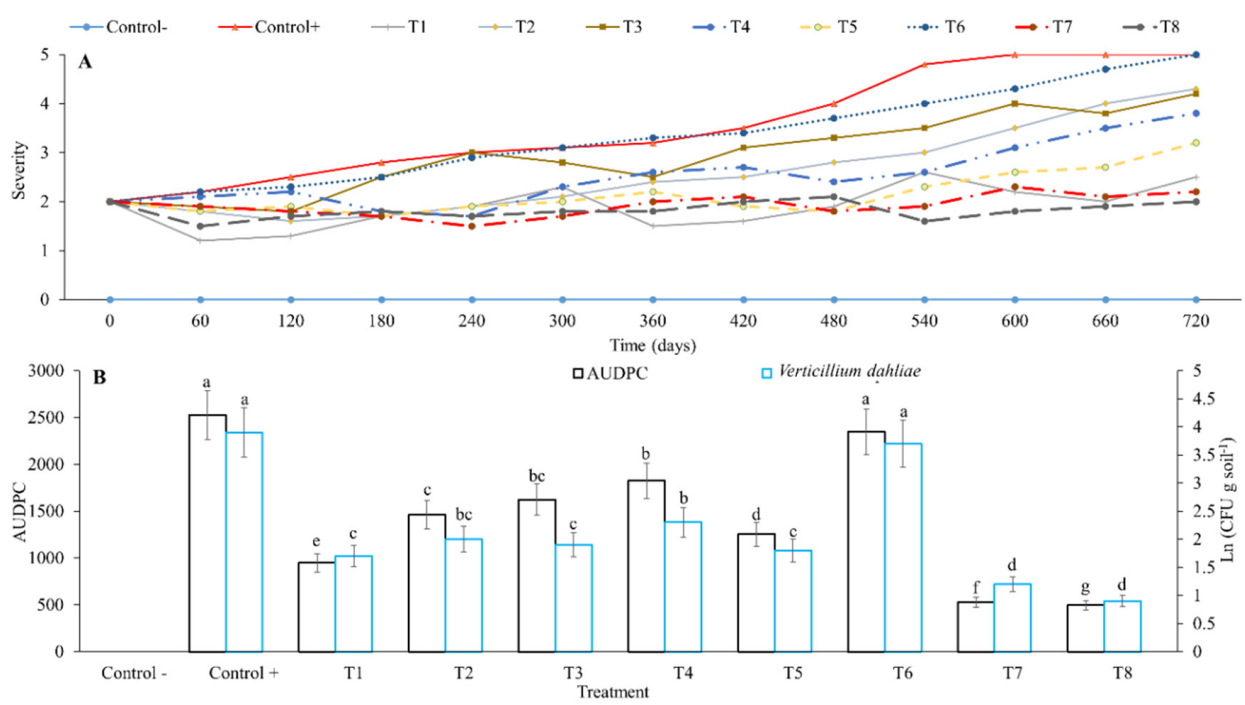

Figure 4. Area under disease progress curve (AUDPC), and inoculum of Verticillium dahliae under management practices of disease on field conditions. (A): Dynamics of the severity of $V$. dahliae under different treatments. (B): AUDPC of $V$. dahliae under different treatments. Error bars represent the confidence interval of the mean, validated by Tukey mean separation test. Equal letters indicate that there are no significant differences $(p>0.05)$. The following treatments were evaluated under field conditions (where "fi" stands for field conditions): fi-healthy control: non-inoculated control of healthy plants; fi-diseased control: plants infected with $V$. dahliae; T1fi (fungicide): Verticillium-infected plants + application of fungicide in drench (benomyl in rotation with azoxystrobin); T2fi (pruning): Verticillium-infected plants + sanitary pruning; T3fi (solarization): Verticillium-infected plants + solarization; T4fi (Trichoderma-mycorrhiza-sucrose): Verticillium-infected plants + soil inoculation with Trichoderma sp. and R. fasciculatum; T5fi (organic matter): Verticillium-infected plants + application of compost; T6fi (drainage): Verticillium-infected plants + a drainage network around the base of the stem; T7fi (pruning-solarization-Trichoderma-mycorrhiza-sucrose-organic matter-drainage): Verticillium-infected plants + integrated management proposal without fungicide consisting of simultaneous application of T2fi + T3fi + T4fi + T5fi + T6fi; and T8fi (fungicide-pruning-solarization-Trichoderma-mycorrhiza-sucrose-organic matter-drainage): Verticillium-infected plants + integrated management proposal with fungicide consisting of simultaneous application of T1fi + T2fi + T3fi + T4fi + T5fi + T6fi. 

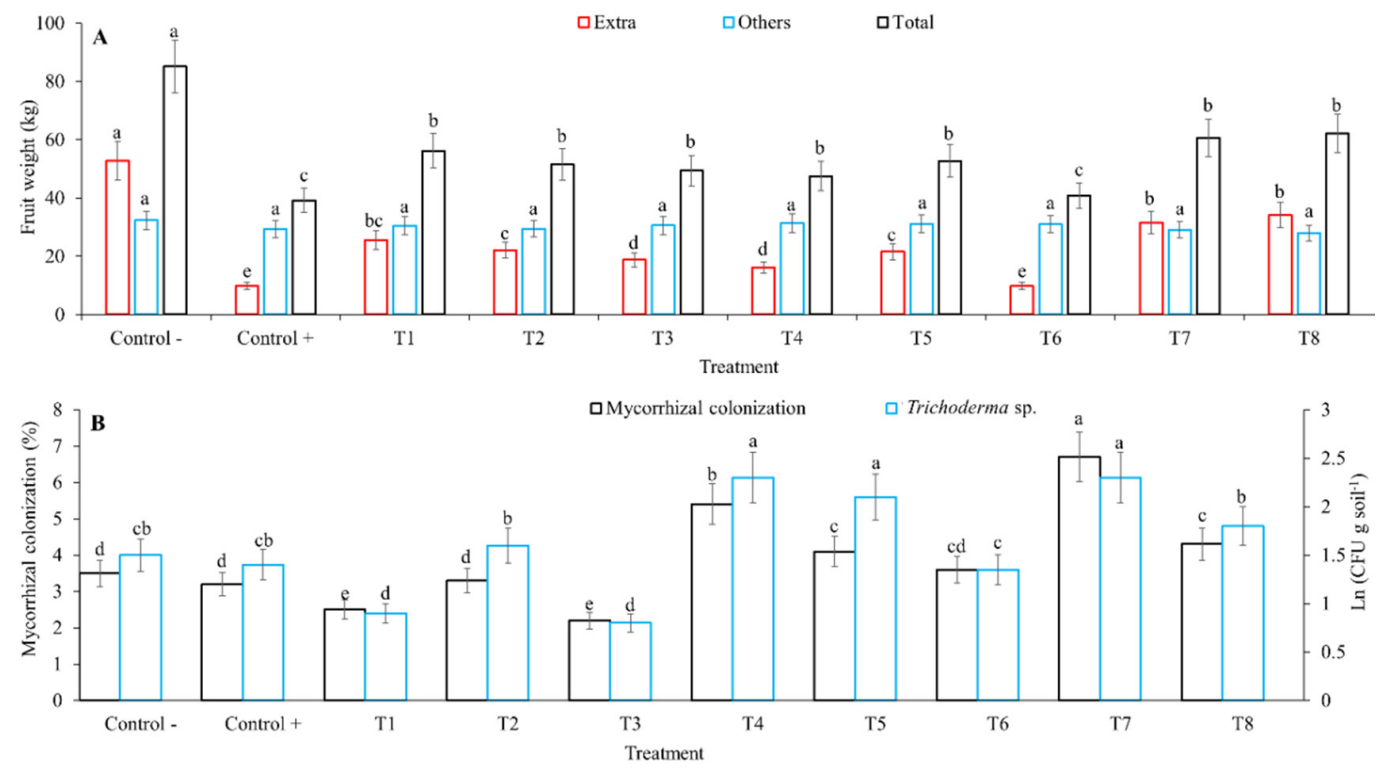

Figure 5. Quantity and quality of fruit production, mycorrhizal colonization, Trichoderma sp. inoculum and in management practices of $V$. dahliae under field conditions. (A): Production and quality of Hass avocado crops under different treatments for $V$. dahliae. (B): mycorrhizal colonization and inoculum of Trichoderma sp. quantified under different treatments of wilt disease. Error bars represent the confidence interval of the mean, validated by Tukey mean separation test. Equal letters indicate that there are no significant differences $(p>0.05)$. The following treatments were evaluated under field conditions (where "fi" stands for field conditions): fi-healthy control: non-inoculated control of healthy plants; fi-diseased control: plants infected with $V$. dahliae; T1fi (fungicide): Verticillium-infected plants + application of fungicide in drench (benomyl in rotation with azoxystrobin); T2fi (pruning): Verticillium-infected plants + sanitary pruning; T3fi (solarization): Verticilliuminfected plants + solarization; T4fi (Trichoderma-mycorrhiza-sucrose): Verticillium-infected plants + soil inoculation with Trichoderma sp. and R. fasciculatum; T5fi (organic matter): Verticillium-infected plants + application of compost; T6fi (drainage): Verticillium-infected plants + a drainage network around the base of the stem; T7fi (pruning-solarization-Trichodermamycorrhiza-sucrose-organic matter-drainage): Verticillium-infected plants + integrated management proposal without fungicide consisting of simultaneous application of T2fi + T3fi + T4fi + T5fi + T6fi; and T8fi (fungicide-pruning-solarizationTrichoderma-mycorrhiza-sucrose-organic matter-drainage): Verticillium-infected plants + integrated management proposal with fungicide consisting of simultaneous application of T1fi + T2fi + T3fi + T4fi + T5fi + T6fi.

\section{Discussion}

Adequate programs of integrated management for Verticillium wilt in avocado are scarce and not readily available $[9,15]$. Here, we identified treatments for avocado wilt caused by $V$. dahliae, which reduced disease, increased the percentage of the high value "extra" fruit category and showed a good cost/benefit relationship (Table 1). Integrated disease management proposals T7fi (sanitary pruning, solarization, Trichoderma sp., R. fasciculatum, a source of energy in the form of sucrose, compost, and drainage without fungicides) and T8fi (same as T7fi, but including benomyl and azoxystrobin fungicides in rotation), consisting of combined strategies, exhibited the best effect in controlling the wilt disease caused by $V$. dahliae in avocado crops (Figures 4 and 5, and Table 1).

Individual control methods as fungicide applications, application of one biocontroller, addition of organic matter or other amendments, usually reduce some level of disease. However, when all those strategies are combined they can act in synergy reducing or suppressing disease development to an effective threshold for farmers. Each strategy has its own advantages and constraints (Figures 4 and 5).

Fungicide effectiveness in wilt control have been shown in other crops such as Capsicum annuum L. cv. Soroksari, where benomyl application reduced Verticillium wilt in 88.2 and $94.6 \%$ when applied after and before infection with the pathogen, respectively [32]. A negative side effect of benomyl application observed in the present work was the reduction of other microorganisms that may be beneficial for avocado crops such as 
Trichoderma sp. and R. fasciculatum (Figure S2). Similarly, the total number of fungi in soil was reduced after several applications of metalxyl + mancozeb, for controlling P. cinnamomi in avocado crops $[16,17]$. In addition, continuous application of chemical fungicides may select resistant isolates in the pathogen population, causing ineffectiveness of the molecule, as has been widely reported for benomyl [42]. Therefore, the use of chemical fungicides may be useful as part of an integrated disease management program, but caution should be taken to prevent aforementioned problems. As T7fi was effective to manage Verticillium wilt disease, it should be preferred to T8fi because no fungicides are included in T7fi (Figures 4 and 5).

The use of microorganisms such as Trichoderma sp. and mycorrhizas for the biological control of diseases caused by Verticillium sp. have proven useful in several crops [21,22]. In addition, mycorrhizas such as R. fasciculatum, have shown positive effects, improving the nutritional status of the host plant, particularly the phosphorus uptake $[19,20]$. However, despite the large amount of literature available about biological control agents against Verticillium, few products effective at the field-level have been developed [1]. An apparent antagonism between Trichoderma sp., and R. fasciculatum was identified in the present work. Antagonism between Trichoderma spp. and mycorrhizas has been observed previously in many plants, including avocado [43,44]. To lessen this problem, the addition of an energy source readily available such as sucrose has been reported as having a positive effect in reducing such antagonism [40]. Further research is required to solve this challenge.

Pruning may help in controlling disease by eliminating inoculum sources and by stimulating regrowth of new disease-free tissues as observed in our research. Besides, solarization reduced the inoculum amount in an apparent temperature-dependent manner (Figure S1B). The temperature for controlling effectively Verticillium sp. has been reported to be above $42{ }^{\circ} \mathrm{C}$ [45]. This finding suggests that it is important to closely monitor temperature during the solarization process and to measure viable pathogen populations, looking for an adequate control of $V$. dahliae inoculum in soil, before plant establishment in nursery or in field conditions. Solarization and heat treatments have been tested in the management of Verticillium with encouraging results [5,10]; therefore, their effects should not be neglected as a component of an integrated management of Verticillium wilt.

Although the drainage itself did not show a clear effect on disease management, its implementation in avocado crops should not be ignored because plant roots are sensitive to oxygen reduction and the water to air ratio and availability of oxygen significantly influences growth and mineral nutrition of avocado plants [46]. In general, it is widely accepted that hypoxia and anoxia are of high importance as abiotic causal agents of the avocado wilt disease complex and the oxygen level in soil plays an important role in the dynamics of populations of pathogenic/beneficial microorganisms in the soil profile $[16,17]$. Specifically, severity of root rot caused by P. cinnamomi is increased under soil flooding/low oxygen conditions [17]. However, it was observed that viable Verticillium microsclerotia was reduced in soil under flooding conditions and severity of Verticillium disease was increased under non-flooding conditions compared to flooding conditions [47], suggesting an additional role of other factors such as edaphoclimatic conditions and the plant genotype on the infection outcome. Therefore, as avocado production may be significantly affected by poor soil oxygen availability, much more research is needed to make accurate conclusions about the relationship between avocado, soil oxygen levels and management of Verticillium wilt. Climate change is expected to strongly influence plant diseases; therefore, further research using precise measurements of available contents of water and oxygen using different drainage methods and edaphoclimatic conditions is a necessary step towards clarification of the role of air-water ratio in the Verticillium avocado wilt disease.

Organic matter is crucial for good soil health, and its beneficial effects for avocado and others crops have been widely reported $[6,14,48]$. Some studied effects include increase in beneficial microbe populations, reduction of pathogenic microbe populations and disease incidence and severity, in addition to reduction of the stress caused by climate change and many others $[6,14,47]$. Currently, the addition of green compost from agricultural 
and industrial waste is reported as one of the most viable economic and environmental alternatives for increasing the beneficial microbiota in soils $[25,26]$. The addition of different sources of organic matter reduced disease severity caused by Verticillium spp. in several crops [23-26,48]. It has been shown that the addition of green compost to soils with high inoculum pressure of soilborne pathogens generates a suppression effect, becoming an excellent strategy to incorporate into an integrated plant management, which include fungi genus Verticillium $[25,26]$. However, organic amendments significantly varied in type, source, chemical components, crop yield, disease control and many other factors that may affect results $[1,25,26]$. Even more, it has been found that in some cases, organic matter reduce Phytophthora root rot, and in others may even increase disease severity [14,49]. Organic amendments are a valuable tool for Verticillium wilt control, but time consuming and expensive efforts on research should be made to find appropriate combinations for each specific planting sites and edaphoclimatic conditions where avocado orchards are grown. However, they significantly reward the effort, as observed in the present research.

Each individual control strategy has shown some extent of effective reduction of Verticilium wilt in several crop systems [1]. Integrated management systems, which include several strategies have been implemented for crops as potato, olive, lettuce, cotton and others $[1,2,5]$. In avocado, research about integrated management of Verticillium is limited compared to other pathogens such as P. cinnamomi [14,31]. The integrated management proposed in the present research proved to be effective in the conditions performed; however, results may vary from location to location due to factors already mentioned, so it is important to make local adaptations to each specific condition because the benefit may exceed the investment on research. Other emerging strategies for Verticillium control as new sources of plant resistance, disinfectants, heat treatments, ways of biological control delivery, and others, may be implemented as they are developed, together with our proposal of integrated management of Verticillium wilt [7,10,23].

Integrated management practices proposed in T7fi and T8fi are economically viable, showing a good cost/benefit relationship, where the increase in the production of fruit in the extra quality category plays an important role because of its high price in the market that compensates and overcomes a lower net production. These results indicate that implementation of an appropriate integrated management for this disease may increase the profit of the avocado plantation. As mentioned above, T7fi should be preferred to T8fi where possible because T7fi does not include fungicides of chemical synthesis. Even more, a similar approach has proved to be effective for the control of P. cinnamomi [14], suggesting that it is possible to design a combined strategy against the two most important pathogens causing avocado wilt.

\section{Conclusions}

The avocado wilt disease caused by $V$. dahliae is economically important in Colombian conditions. Integrated management may reduce the negative effects and economic losses caused by this pathogen by increasing the quality of fruits that get the higher prices. No single control method for Verticillium is completely effective, so implementation of several compatible strategies adapted to each local condition, together with a careful economic analysis, should be performed to satisfactorily manage this disease while keeping reasonable profits. The present work is an important contribution to the avocado industry because wilt symptoms caused by $V$. dahliae are easily confused with those caused by other causal agents, inducing erroneous diagnostics and implementation of ineffective control methods that may induce an increase in costs of production and even larger losses.

Supplementary Materials: The following are available online at https: / www.mdpi.com/article / 10.3390 /agronomy11101932/s1, Table S1: Edaphic variables of the soil in the crop plot and laboratory tests, Table S2: Description of experiments under net-house conditions, Table S3: Supporting information 65. Description of experiments under field conditions, Figure S1: (A): climatic conditions in the plots evaluated during the years 2012-2014. (B): dynamics of temperature associated with 
solarization under net-house and field conditions, Figure S2: Effect of application of fungicides to soil in microbial beneficial populations, Figure S3: Scheme of drainage treatment under field conditions.

Author Contributions: Conceptualization, J.G.R.-G. and J.G.M.-O.; formal analysis, J.G.R.-G.; investigation, J.G.R.-G. and J.G.M.-O.; writing-original draft preparation, J.G.M.-O. and J.G.R.-G.; writing-review and editing, J.G.R.-G. and J.G.M.-O.; supervision, J.G.R.-G. and J.G.M.-O. All authors have read and agreed to the published version of the manuscript.

Funding: This research received no external funding.

Institutional Review Board Statement: Not applicable, our study did not involve humans or animals.

Informed Consent Statement: Not applicable, our study did not involve humans.

Data Availability Statement: The data that support the findings of this study are available from the corresponding author upon reasonable request.

Acknowledgments: To “Universidad Nacional de Colombia sede Medellín” that provided partial funding, and Colciencias that provided the Ph. D. scholarship funding of J.G.R.-M. We are grateful with avocado producers for valuable information and help during research, Guillermo Noreña in North region, and Fernando Gomez in East region of Antioquia, Colombia. All authors have approved the final article. This research did not receive any specific grant from funding agencies in the public, commercial, or not-for-profit sectors. The funding source had no involvement in study design; in the collection, analysis and interpretation of data; in the writing of the report; and in the decision to submit the article for publication.

Conflicts of Interest: The authors declare no conflict of interest.

\section{References}

1. Klosterman, S.J.; Atallah, Z.K.; Vallad, G.E.; Subbarao, K.V. Diversity, pathogenicity, and management of Verticillium species. Annu. Rev. Phytopathol. 2009, 47, 39-62. [CrossRef]

2. Pegg, G.; Brady, B. Verticillium Wilts; CABI Publishing: New York, NY, USA, 2002.

3. Bhat, R.G.; Subbarao, K.V. Host Range specificity in Verticillium dahliae. Phytopathology 1999, 89, 1218-1225. [CrossRef] [PubMed]

4. Inderbitzin, P.; Davis, R.M.; Bostock, R.M.; Subbarao, K.V. Identification and differentiation of Verticillium species and V. longisporum lineages by simplex and multiplex PCR assays. PLoS ONE 2013, 8, e65990. [CrossRef]

5. Carroll, C.L.; Carter, C.A.; Goodhue, R.E.; Lawell, C.-Y.C.L.; Subbarao, K.V. A Review of control options and externalities for Verticillium wilts. Phytopathology 2018, 108, 160-171. [CrossRef]

6. Papasotiriou, F.G.; Varypatakis, K.G.; Christofi, N.; Tjamos, S.E.; Paplomatas, E.J. Olive mill wastes: A source of resistance for plants against Verticillium dahliae and a reservoir of biocontrol agents. Biol. Control 2013, 67, 51-60. [CrossRef]

7. Gómez-Gálvez, F.-J.; Rodríguez-Jurado, D. Potential wfficacy of soil-applied disinfectant treatments against Verticillium wilt of olive. Crop Prot. 2018, 106, 190-200. [CrossRef]

8. Pegg, G.F. The impact of Verticillium diseases in agriculture. Phytopathol. Mediterr. 1984, 23, 176-192.

9. Zentmyer, G. Avocado diseases. Trop. Pest Manag. 1984, 30, 677-682. [CrossRef]

10. Morello, P.; Díez, C.M.; Codes, M.; Rallo, L.; Barranco, D.; Trapero, A.; Moral, J. Sanitation of olive plants infected by Verticillium dahliae using heat treatments. Plant Pathol. 2016, 65, 412-421. [CrossRef]

11. Zentmyer, G. Verticillium wilt of avocado. Phytopathology 1949, 39, 677-682.

12. Ramírez-Gil, J.G.; Morales, J.G. Polyphasic Identification of preharvest pathologies and disorders in avocado cv. Hass. Agron. Colomb. 2019, 37, 213-227. [CrossRef]

13. Bubici, G.; Amenduni, M.; Colella, C.; D'Amico, M.; Cirulli, M. Efficacy of acibenzolar-s-methyl and two strobilurins, azoxystrobin and trifloxystrobin, for the control of corky root of tomato and Verticillium wilt of wggplant. Crop Prot. 2006, 8, 814-820. [CrossRef]

14. Ramírez-Gil, J.G.; Morales-Osorio, J.G. Integrated proposal for management of root rot caused by Phytophthora cinnamomi in avocado vv. Hass crops. Crop Prot. 2020, 137, 105271. [CrossRef]

15. Haberman, A.; Tsror (Lahkim), L.; Lazare, S.; Hazanovsky, M.; Lebiush, S.; Zipori, I.; Busatn, A.; Simenski, E.; Dag, A. Management of Verticillium wilt of avocado using tolerant rootstocks. Plants 2020, 9, 531. [CrossRef] [PubMed]

16. Ramírez-Gil, J.G.; Henao-Rojas, J.C.; Morales-Osorio, J.G. Mitigation of the adverse wffects of the El Niño (El Niño, La Niña) Southern Oscillation (ENSO) phenomenon and the most important diseases in avocado cv. Hass crops. Plants 2020, 9, 790. [CrossRef] [PubMed]

17. Ramírez-Gil, J. Avocado wilt complex disease, implications and management in colombia. Rev. Fac. Nac. Agron. Medellín 2018, 71, 8525-8541. [CrossRef]

18. Ramírez-Gil, J.G.; Peterson, A.T. Current and potential distributions of most important diseases affecting Hass avocado in Antioquia Colombia. J. Plant Prot. Res. 2019, 59, 214-228. [CrossRef] 
19. Plassard, C.; Becquer, A.; Garcia, K. Phosphorus transport in mycorrhiza: How far are we? Trends Plant Sci. 2019, $24,794-801$. [CrossRef]

20. Sieverding, E.; da Silva, G.A.; Berndt, R.; Oehl, F. Rhizoglomus, a New Genus of the Glomeraceae. Available online: https: //www.ingentaconnect.com/content/mtax/mt/2015/00000129/00000002/art00017 (accessed on 19 November 2018).

21. Deketelaere, S.; Tyvaert, L.; França, S.C.; Höfte, M. Desirable traits of a good biocontrol agent against Verticillium wilt. Front. Microbiol. 2017, 8, 1186. [CrossRef]

22. Yuan, Y.; Feng, H.; Wang, L.; Li, Z.; Shi, Y.; Zhao, L.; Feng, Z.; Zhu, H. Potential of endophytic fungi isolated from cotton roots for biological control against Verticillium wilt disease. PLoS ONE 2017, 12, e0170557. [CrossRef]

23. Larkin, R.P.; Honeycutt, C.W.; Olanya, O.M. Management of Verticillium wilt of potato with sisease-suppressive green, manures and as affected by previous cropping history. Plant Dis. 2011, 95, 568-576. [CrossRef]

24. Markakis, E.A.; Fountoulakis, M.S.; Daskalakis, G.C.; Kokkinis, M.; Ligoxigakis, E.K. The Suppressive effect of compost amendments on Fusarium Oxysporum f.Sp. Radicis-Cucumerinum in cucumber and Verticillium dahliae in eggplant. Crop Prot. 2016, 79, 70-79. [CrossRef]

25. De Corato, U.; Patruno, L.; Avella, N.; Lacolla, G.; Cucci, G. Composts from green sources show an increased suppressiveness to soilborne plant pathogenic fungi: Relationships between physicochemical properties, disease suppression, and the microbiome. Crop Prot. 2019, 124, 104870. [CrossRef]

26. De Corato, U.; Salimbeni, R.; de Pretis, A.; Patruno, L.; Avella, N.; Lacolla, G.; Cucci, G. Microbiota from 'next-generation green compost' improves suppressiveness of composted municipal-solid-waste to soil-borne plant pathogens. Biol. Control 2018, 124, 1-17. [CrossRef]

27. Rekanovic, E.; Potocnik, I.; Milijasevic-Marcic, S.; Stepanovic, M.; Todorovic, B.; Mihajlovic, M. Efficacy of seaweed concentrate from Ecklonia maxima (Osbeck) and conventional fungicides in the control of Verticillium wilt of pepper. Pestic. Fitomed. 2010, 25, 319-324. [CrossRef]

28. Trade Map_Trade Statistics for International Business Development. Available online: https://www.trademap.org/Index.aspx (accessed on 31 August 2021).

29. Aguacate I Hass I Corpohass I. Available online: https://www.corpohass.com/ (accessed on 31 August 2021).

30. FAO FAOSTAT. 2021. Available online: http:/ /www.fao.org/faostat/es/\#home (accessed on 9 January 2021).

31. Tamayo, P. Enfermedades del aguacate. Politecnica 2007, 4, 51-70.

32. Rekanovic, E.; Milijasevic, S.; Todorovic, B.; Potocnik, I. Possibilities of biological and chemical control of Verticillium wilt in pepper. Phytoparasitica 2007, 35, 436. [CrossRef]

33. Thorat, S.; More, B.; Konde, B. Chemical control of Verticillium wilt of eggplant (Solanum melongena L.) by soil and plant application. Hind. Antibiot. Bull. 1976, 18, 117-118.

34. Madden, L.V.; Hughes, G.; van den Bosch, F. The Study of Plant Disease Epidemics; American Phytopathological Society (APS Press): St. Paul, MN, USA, 2007; ISBN 978-0-89054-505-8.

35. Ramírez-Gil, J.G.; Morales-Osorio, J.G.; Ramírez-Gil, J.G.; Morales-Osorio, J.G. Development and validation of severity scales of avocado wilt complex caused by Phytophthora cinnamomi, Verticillium dahliae and hypoxia-anoxia disorder and their physiological responses in avocado plants. Agron. Colomb. 2020, 38, 85-100. [CrossRef]

36. Goud, J.C.; Termorshuizen, A.J. Quality of methods to quantify microsclerotia of Verticillium dahliae in soil. Eur. J. Plant Pathol. 2003, 109, 523-534. [CrossRef]

37. Giovannetti, M.; Mosse, B. An Evaluation of techniques for measuring vesicular arbuscular mycorrhizal infection in roots. New Phytol. 1980, 84, 489-500. [CrossRef]

38. Phillips, J.M.; Hayman, D.S. Improved procedures for clearing roots and staining parasitic and vesicular-arbuscular mycorrhizal fungi for rapid assessment of infection. Trans. Br. Mycol. Soc. 1970, 55, 158-IN18. [CrossRef]

39. Kormanik, P.P.; Bryan, W.C.; Schultz, R.C. Procedures and equipment for staining large numbers of plant root samples for endomycorrhizal assay. Can. J. Microbiol. 1980, 26, 536-538. [CrossRef]

40. Ramirez-Gil, J.; Osorno, L.; Morales Osorio, J.G.; Osorio, N. Addition of glucose as carbon source improves the Glomus fasciculatumTrichoderma harzianum-Leucaena leucocephala interactions. In Proceedings of the XV National Congress of Biotechnology and Bioengineering and 12 th International Symposium on the Genetics of Industrial Microorganisms (GIM 2013), Cancun, Mexico, 23-28 June 2013.

41. Vilariño, A.; Sainz, M.J. Treatment of Glomus mosseae propagules with $50 \%$ sucrose increases spore germination and inoculum potential. Soil Biol. Biochem. 1997, 29, 1571-1573. [CrossRef]

42. Locke, T.; Thorpe, I. Benomyl tolerance in Verticillium dahliae Kleb. Plant Pathol. 1997, 25, 152. [CrossRef]

43. Castillo, A.G.; Puig, C.G.; Cumagun, C.J.R. Non-synergistic effect of Trichoderma harzianum and Glomus spp. in reducing infection of Fusarium wilt in banana. Pathogens 2019, 8, 43. [CrossRef]

44. Martinez, A.; Obertello, M.; Pardo, A.; Ocampo, J.A.; Godeas, A. Interactions between Trichoderma pseudokoningii strains and the arbuscular mycorrhizal fungi Glomus mosseae and Gigaspora rosea. Mycorrhiza 2004, 14, 79-84. [CrossRef] [PubMed]

45. Pullman, G.S.; DeVay, J.E.; Garber, R. Soil solarisation and thermal death: A logarithmic relationship between time and temperature for four soilborne plant pathogens. Phytopathology 1981, 71, 959-964. [CrossRef]

46. Gil, P.M.; Bonomelli, C.; Schaffer, B.; Ferreyra, R.; Gentina, C. Effect of soil water-to-air ratio on biomass and mineral nutrition of avocado trees. J. Soil Sci. Plant Nutr. 2012, 12, 609-630. [CrossRef] 
47. Short, D.P.G.; Sandoya, G.; Vallad, G.E.; Koike, S.T.; Xiao, C.-L.; Wu, B.-M.; Gurung, S.; Hayes, R.J.; Subbarao, K.V. Dynamics of Verticillium species microsclerotia in field soils in response to fumigation, cropping patterns, and flooding. Phytopathology 2015, 105, 638-645. [CrossRef]

48. Bonilla, N.; Cazorla, F.M.; Martínez-Alonso, M.; Hermoso, J.M.; González-Fernández, J.J.; Gaju, N.; Landa, B.B.; de Vicente, A. Organic amendments and land management affect bacterial community composition, diversity and biomass in avocado crop soils. Plant Soil 2012, 357, 215-226. [CrossRef]

49. Downer, J.; Faber, B.; Menge, J. Factors affecting root rot control in mulched avocado orchards. HortTechnology 2002, 12, 601-605. [CrossRef] 